\title{
Pseudoquiste de Morel-Lavallée tras abdominoplastia sin lipoaspiración
}

\section{Morel-Lavallee pseudocyst after ahdominoplasty without liposuction}

\author{
Fernández García, A.*, Fernández Pascual, C.**, Santoyo Gil-López, F.**, \\ Alonso Rosa, S. ${ }^{* 1 *}$
}

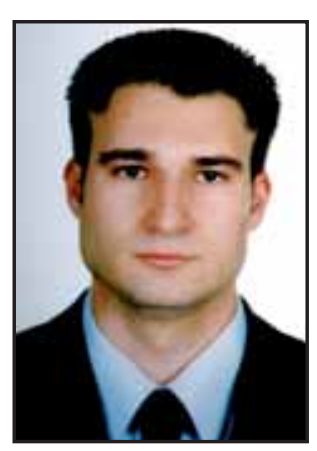

Fernández García, A.

\section{Resumen}

El médico francés Morel-Lavallée describió en 1853 la formación de pseudoquistes secundarios a traumatismos tangenciales con separación de los planos subcutáneo y fascial en miembros inferiores. La dermolipectomía abdominal se ha desarrollado como un procedimiento muy eficaz y satisfactorio, especialmente combinado con lipoaspiración local. Esta técnica puede producir necrosis grasa, seroma y extravasación de Morel-Lavallee. Aunque infrecuente, este fenómeno puede ocurrir en abdominoplastias incluso sin liposucción. En este trabajo presentamos una paciente de 67 años de edad que desarrolló un pseudoquiste de Morel-Lavallée de 570 gr, 2 años después de una dermolipectomía abdominal sin lipoaspiración asociada. Se revisan los procedimientos diagnósticos y opciones terapéuticas.

Palabras clave $\begin{aligned} & \text { Morel-Lavallée, Pseudoquiste, Abdomi- } \\ & \text { noplastia }\end{aligned}$
Código numérico 5311

\begin{tabular}{|ll|}
\hline Key words & $\begin{array}{l}\text { Morel-Lavallée, Pseudocyst, Abdomino- } \\
\text { plasty }\end{array}$ \\
Numeral Code & 5311
\end{tabular}

The french physician Morel-Lavallée described in 1853 the formation of pseudocysts after tangential traumas with separation of the subcutaneous and fascial layers in the lower limbs. Abdominoplasty has evolved as a very effective and satisfactory procedure, specially when combined with local liposuction. This technique can produce fat necrosis, seroma and Morell-Lavallée extravasations. Although not very common, this phenomenon can occur following an abdominoplasty even without liposuction. In this report we present a 67 year old woman who developed a pseudocyst of $570 \mathrm{gr}, 2$ years after an abdominoplasty without liposuction. We review the diagnostic procedures and the treatment options. 


\section{Introducción}

Las complicaciones secundarias a dermolipectomía abdominal son variadas y de diferente gravedad. Los tromboembolismos, atelectasias, infecciones, necrosis cutánea, retardo cicatricial, hematomas y seromas son algunos de los ejemplos más característicos de este tipo de intervención (1). De todas ellas, la más frecuente es el seroma, que llega a aparecer hasta en el 5\% de los casos (2). El sobrepeso y la obesidad son factores de riesgo para el desarrollo de seromas tras la realización de abdominoplastia y lipoaspiración abdominal (3). La formación de seroma, hematoma o necrosis grasa en el plano de disección de la pared abdominal tras una dermolipectomía con lipoaspiración, puede condicionar la aparición de extravasación de Morel-Lavallée (4).

El médico francés Morel-Lavallée describió en 1853 la formación de pseudoquistes secundarios a traumatismos tangenciales con separación de los planos subcutáneo y fascial en miembros inferiores. Esta complicación ha sido comunicada con una incidencia en torno al $1 \%$ en abdominoplastias asociadas a lipoaspiración (5) y es aún más frecuente en abdominoplastias sin lipoaspiración combinada (6).

La necrosis grasa y los espacios muertos que generan los túneles de liposucción, facilitan la formación de este tipo de colecciones encapsuladas. No obstante, las abdominoplastias simples pueden generar un plano de deslizamiento entre el colgajo cutáneo y la fascia abdominal que de lugar a pseudoquistes de gran tamaño. Estas formaciones requieren un diagnóstico clínico y radiológico precoz, así como una exéresis completa para su resolución.

\section{CASO CLINICO}

Mujer de 67 años de edad, alérgica a los contrastes yodados, obesa con Indice de Masa Corporal (IMC) 41, diabética tipo I insulinodependiente y con antecedentes de hernia hiatal, ooforectomía por quiste ovárico, apendicectomía y amigdalectomía previas; estenosis de canal lumbar L4-L5.

En 2004 consulta por lipodistrofia y dermocalasia abdominal por lo que es intervenida quirúrgicamente bajo anestesia general para practicarle dermolipectomía abdominal transversa baja con resección vertical infraumbilical y trasposición umbilical. En el postoperatorio se dejaron dos drenajes aspirativos por contraincisión y en la herida se hizo cierre por planos de los colgajos cutáneos. La pieza del faldón abdominal extirpado pesó de 5942 gr; dicha pieza no mostró alteraciones histológicas.

Durante el postoperatorio inmediato la paciente mantuvo presoterapia con faja abdominal durante 2 meses y tratamiento antibiótico con Ciprofloxacino 400gr vía intravenosa cada 12 horas durante 12 días. Presentó débito a través de los drenajes de unos 100 cc al día, de contenido serohemático, motivo por el cual se mantuvieron los drenajes durante 15 días hasta que el débito fue escaso. Una vez retirados, la paciente consultó por notar fluctuación en el plano de disección. Ante la sospecha clínica de seroma coleccionado se realizaron punciones evacuadoras semanales en 2 ocasiones, en cada una de las cuales se extrajeron 50 cc de contenido seroso. En ningún momento apareció fiebre o algún otro síntoma y el seroma se consideró clínicamente resuelto.

Dos años más tarde la paciente consulta por masa abdominal suprapúbica dolorosa a la palpación, de 3 meses de evolución. Durante la exploración se objetiva un abdomen globuloso, blando y depresible y se constata la presencia de un empastamiento suprapúbico de características dolorosas, que se prolonga hacia ambas crestas ilíacas. Una ecografía abdominal reveló la presencia de una masa bien delimitada de ecoestructura heterogénea, alojada en la pared abdominal infraumbilical.

Se solicita una Resonancia Nuclear magnética (RNM)de pared abdominal con gadolinio D.T.P.A en planos axiales y sagitales. Mediante secuencias SE T1 y FSE T2 se pudo visualizar una lesión voluminosa situada en un plano anterior a los rectos abdominales y al oblicuo externo (Fig. 1A), con localización suprapúbica y dimensiones de $8 \times 6 \times 21 \mathrm{~cm}$. La masa presentaba señal intermedia en secuencias T2 y buena delimitación respecto a los planos vecinos (Fig. 1B). La PAAF resultó negativa para células neoplásicas.

Ante la sospecha de pseudoquiste de Morel-Lavallée la paciente fue reintervenida bajo anestesia general: a través de la cicatriz de la incisión suprapúbica previa se disecó el plano subcutáneo hallado una cápsula fibrosa bien delimitada (Fig. 2A) que fue individualizada encontrando una formación quística de $570 \mathrm{~g}$, de forma elíptica con eje mayor transversal, situada en un plano superficial a la aponeurosis del oblicuo externo y de los rectos abdominales, de localización suprapúbica e infraumbilical (Fig. 2 B,C).

Una vez extirpada la pieza se realizó hemostasia y se dejaron 2 drenajes aspirativos por contraincisión. La pared abdominal se cerró por planos. El primer plano consistió en puntos simples de Polyglactin 2/0 aplicados en sentido cráneo-caudal anclando el colgajo a la fascia abdominal. Con esta maniobra se pretende una correcta distribución de la tensión en el colgajo minimizando espacios muertos y deslizamientos. Un segundo plano de sutura sirve de anclaje del tejido subdérmico del colgajo al borde caudal de la herida; empleamos en esta sutura puntos invertidos de 

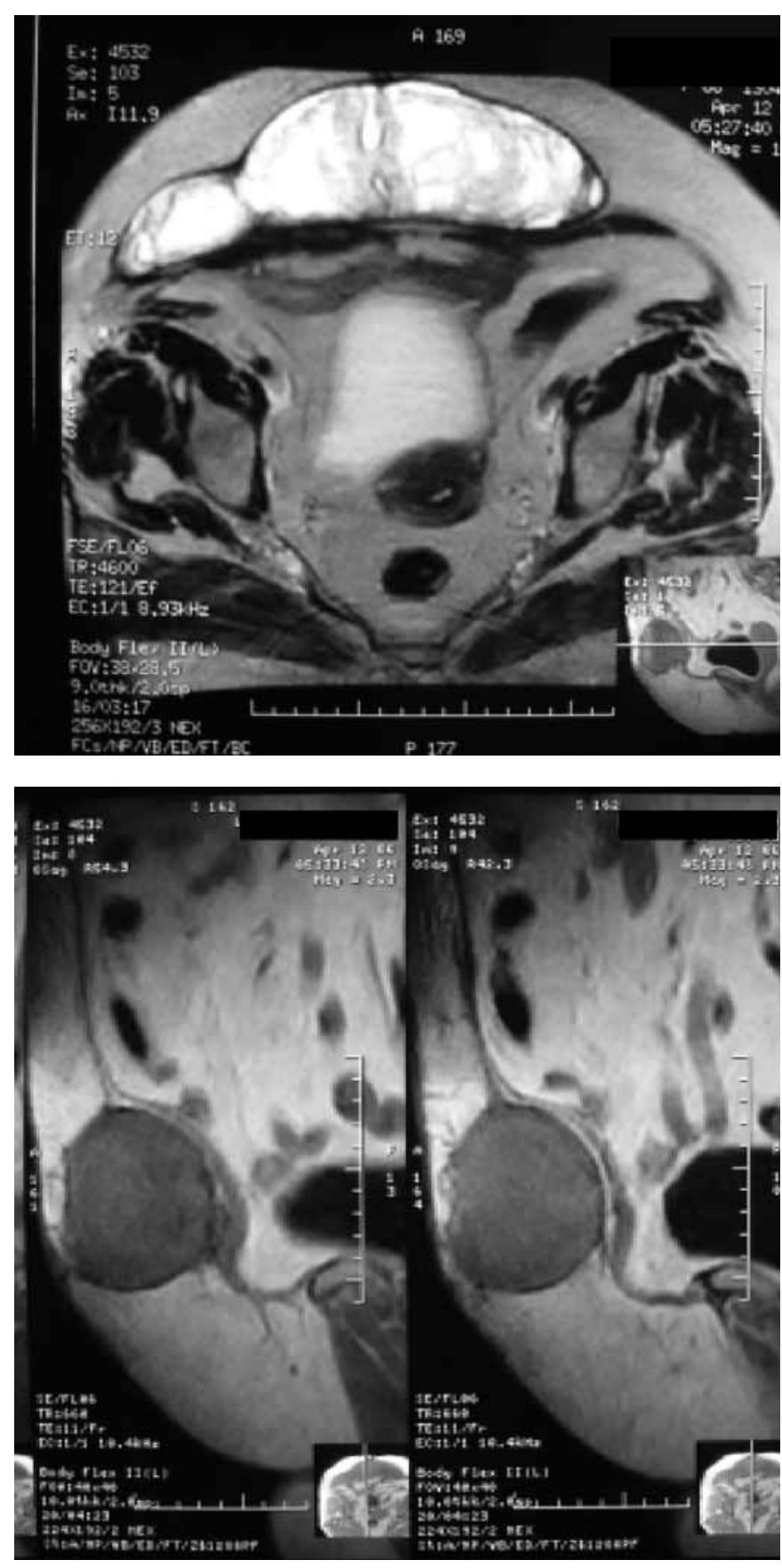

Fig. 1: Resonancia Nuclear Magnética. A: Corte transversal suprapúbico. Masa hiperintensa de contenido heterogéneo bien delimitado de localización suprafascial. B: Corte sagital y medio. Masa de señal intermedia en secuencias T2. Impronta en la línea alba.

Polyglactin 3/0. Por último realizamos una sutura intradérmica con PDS ${ }^{\circledR}$ de $3 / 0$ en plano cutáneo.

Durante y tras la cirugía la paciente recibió tratamiento antibiótico con Ciprofloxacino 400 gr vía intravenosa cada 12 horas y presoterapia con faja abdominal. Los drenajes presentaron débito de unos 100 a $150 \mathrm{cc}$ diarios, de contenido sero-hemático. El ingreso hospitalario se prolongó por este motivo durante 10 días y los drenajes se retiraron a las 3 semanas.

Dos años después de la exéresis del pseudoquiste, la paciente no ha presentado nuevas complicaciones. El contorno abdominal no es muy diferente del que
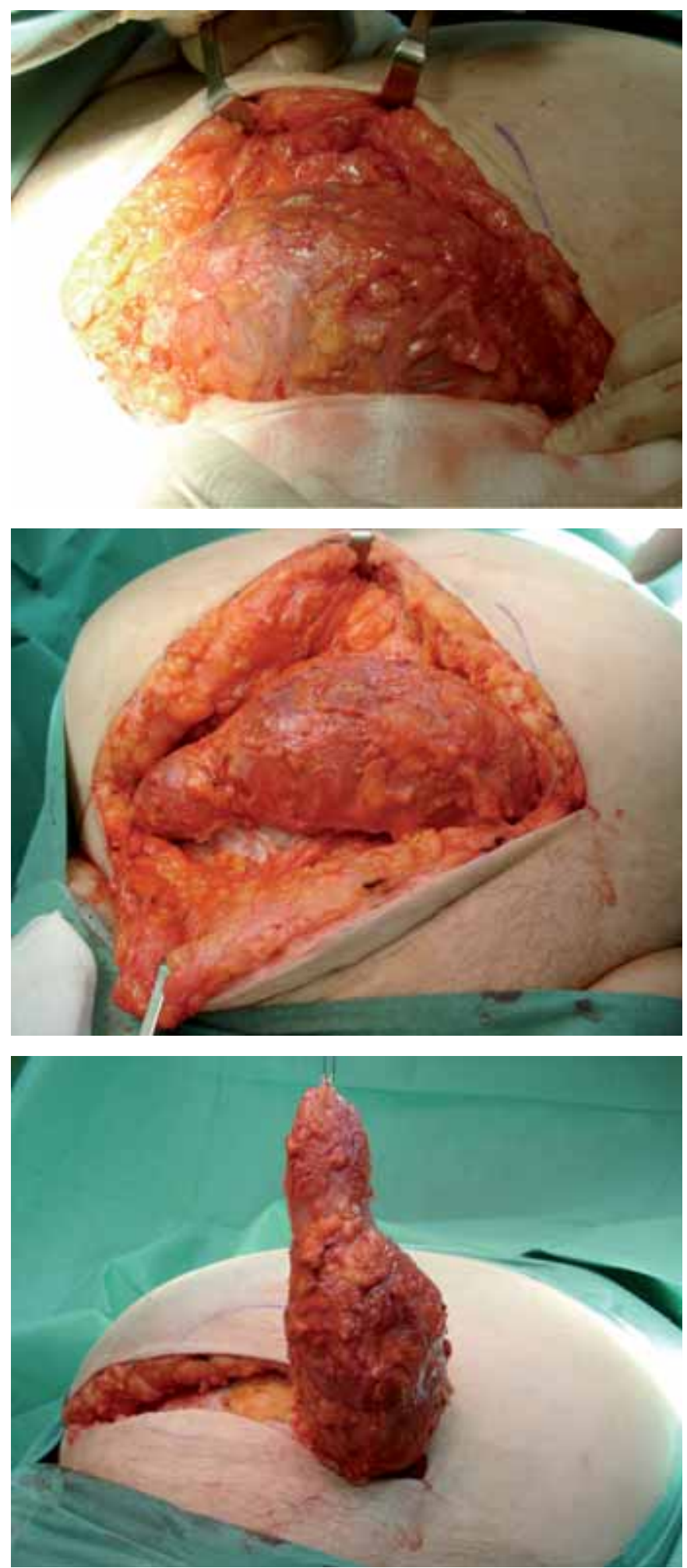

Fig. 2: Imágenes intraoperatorias. A: Abordaje a través de cicatriz de dermolipectomía previa. B: Cápsula fibrosa bien delimitada. C: Disección completa del pseudoquiste.

presentaba antes de la extirpación del pseudoquiste, puesto que éste era sólo detectable a la palpación.

El examen anatomopatológico de la pieza de resección describió una tumoración ovalada de 19,8 x 8 x $5,5 \mathrm{~cm}$ de diámetro y $570 \mathrm{gr}$ de peso (Fig. $3 \mathrm{~A}, \mathrm{~B}$ ), correspondiente a una formación de aspecto quístico con pared fibrosa en periferia de escaso tejido adiposo. Al corte mostraba un espesor parietal de $0,3 \mathrm{~cm} \mathrm{y}$ 

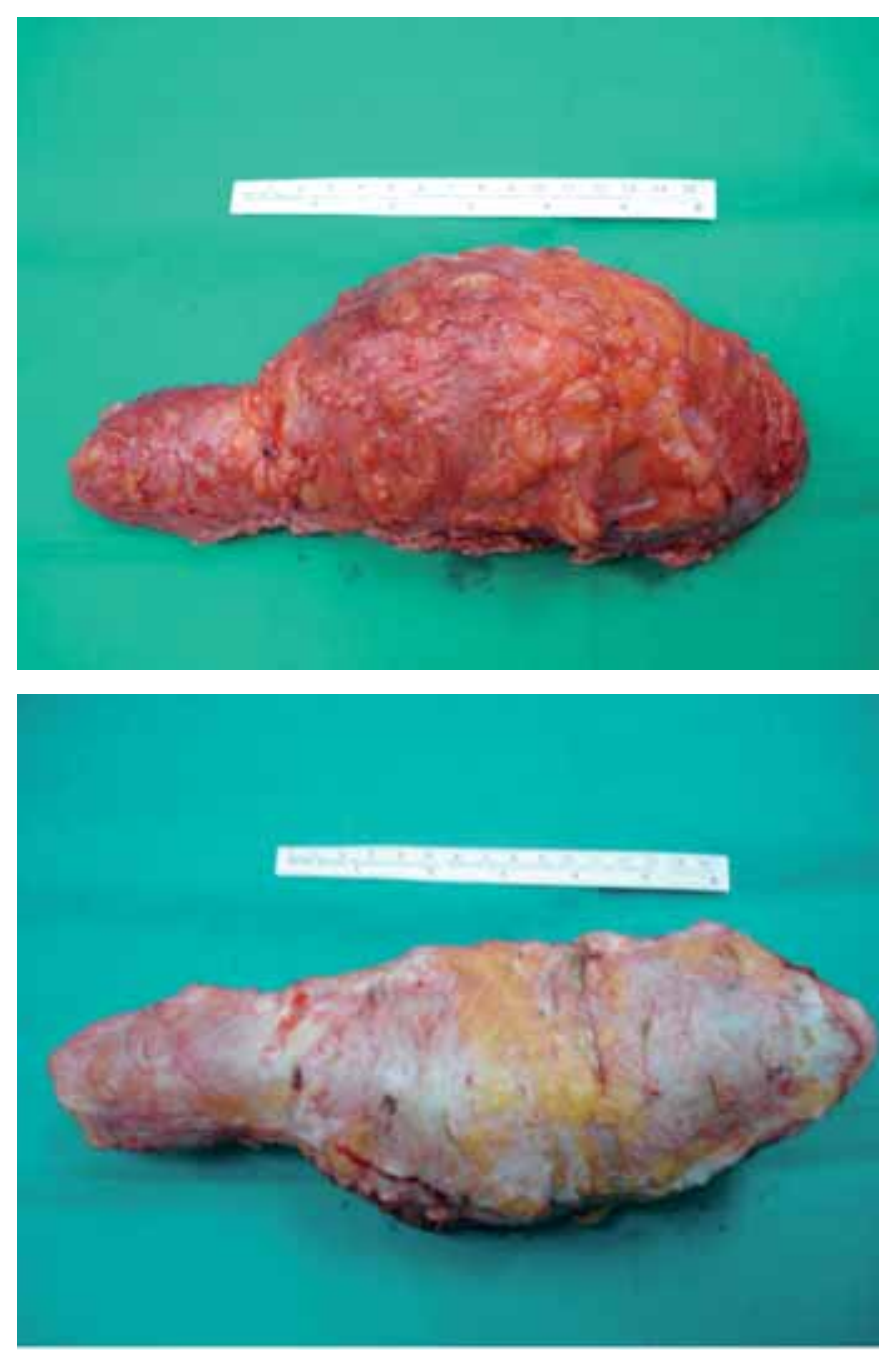

Fig. 3: Pieza de resección. Pseudoquiste de Morel-Lavallée. Dimensiones: 19,8 × 8 × 5,5 cm y 570 gr de peso. A: Faceta anterior. B: Faceta posterior.

estaba ocupada por un material fibrinoso pardo claro, a veces rojizo, del que emanaba en algunos sectores un líquido seroso amarillento con áreas microquísticas y macroquísticas. Al examen microscópico se trataba de una formación pseudoquística constituida por una pared fibrosa sin revestimiento epitelial y ocupada en su luz por material hemático y fibrina en vías de organización (Fig. 4A). Algunos sectores periféricos presentaban histiocitos espumosos así como espacios ópticamente vacíos correspondientes a cristales de colesterol (Fig. 4B).

\section{Discusión}

La formación de pseudoquistes de Morell-Lavallée ha sido clásicamente asociada a traumatismos cerrados de miembro inferior (7). Las fuerzas de deslizamiento tangenciales a un plano óseo, especialmente a nivel de la rodilla o el trocánter mayor, cizallan los tejidos subcutáneos y originan una cavidad virtual suprafascial (8) a la que abocan multitud de vasos sanguíneos y linfáticos seccionados, así como adipo-

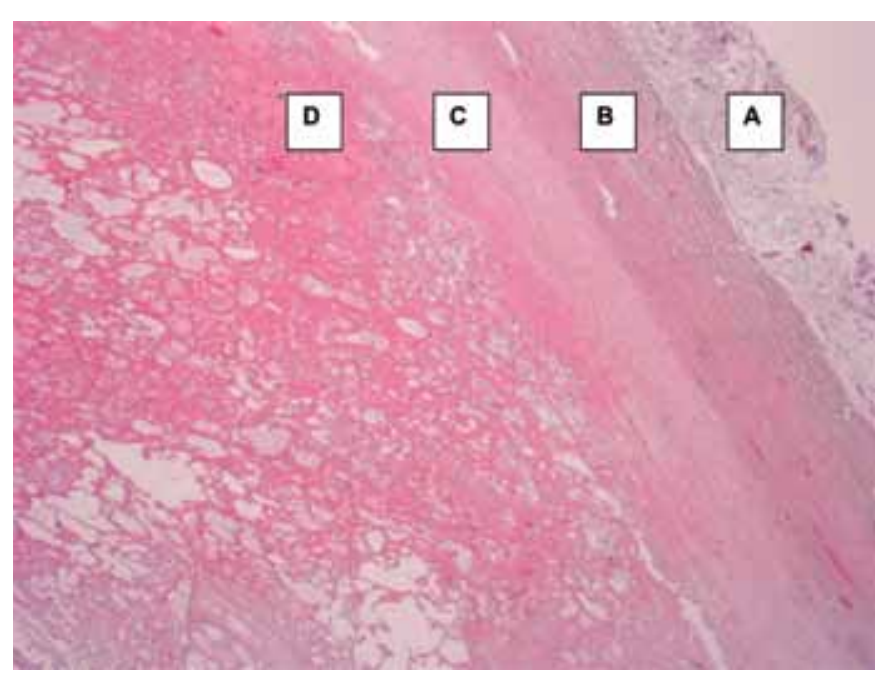

Fig. 4 A: Imagen anatomopatológica de la pieza de resección- Corte histológico de la pared de la pieza $25 X$, tinción hematoxilina-eosina ( $H$ E). Pueden verse los diferentes estratos del pseudoquiste: A) Tejido hipodérmico. B) Cápsula fibrosa. C) Pared del pseudoquiste "ausencia de revestimiento epitelial". D) Contenido fibrinoso y hemático.

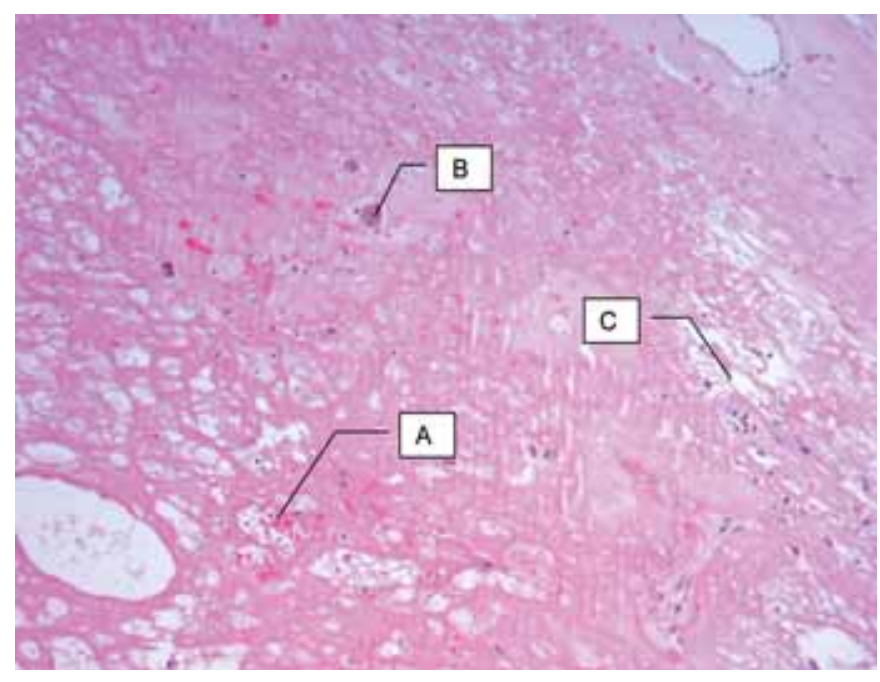

Fig. 4 B: Corte histológico de la pared de la pieza $50 \mathrm{X}$, tinción hematoxilina-eosina (H-E). Se observa material hemático y fibrina en vías de organización. A: Restos hemáticos. B: Histiocitos espumosos "Hemosiderófagos". C: Espacios ópticamente vacíos correspondientes a cristales de colesterol.

citos traumatizados que dan lugar a un acúmulo serohemático en su interior (9). Una situación análoga tiene lugar en las dermolipectomías abdominales, en las que está descrita la formación de presudoquistes de Morell-Lavallée especialmente vinculados a lipoaspiración combinada $(1,4,10)$. Sin embargo son escasas las referencias a esta rara complicación en pacientes sometidos a dermolipectomía simple (6).

En pacientes con un IMC elevado son más frecuentes los seromas tras la realización de una abdominoplastia. En el caso que presentamos, dado que la paciente presentaba patologías asociadas tipo diabetes, obesidad y alergia al yodo, decidimos prolongar la estancia hospitalaria postoperatoria tras la primera intervención. Habitualmente mantenemos ingresados a los pacientes durante 4-5 días, salvo complicaciones; en el caso que nos ocupa, la estancia fue de 12 
días, tiempo condicionado por sus antecedentes, por el control de la glucemia y por el débito de los drenajes y durante el cual se mantuvo la antibioticoterapia por vía intravenosa.

Durante el cierre de los colgajos, es necesario minimizar los espacios muertos y evitar planos de deslizamiento respecto de la fascia abdominal.

Ante una masa en la pared abdominal de características dolorosas tras una dermolipectomía, debe sospecharse la formación de un pseudoquiste; en nuestro caso, seguramente se mantuvo un seroma subclínico tras la primera intervención. En el postoperatorio se produjo el encapsulamiento del mismo, lo que pudo ser la causa de su manifestación clínica tardía como masa dolorosa.

Una ecografía abdominal puede orientar el diagnóstico inicial, que se complementará con una RNM para valorar su seguimiento. En nuestro caso, el diagnóstico del primer seroma fue exclusivamente clínico, puesto que era detectable por palpación. Tampoco se hizo ninguna comprobación de laboratorio del líquido evacuado, puesto que no era purulento ni sospechoso.

Este tipo de pseudoquistes suelen presentarse como colecciones bien delimitadas de contenido heterogéneo, situadas por delante de la fascia abdominal (10).

La aplicación de suturas escalonadas en el cierre de los colgajos abdominales es un recurso eficaz a la hora de prevenir las complicaciones locales como seroma, hematoma y necrosis cutánea en la dermolipectomía abdominal (11). Esta sutura debe ser un anclaje mediante puntos aplicados en sentido cráneocaudal desde el colgajo hacia el borde caudal de la herida, hasta que pueda hacerse el cierre final sin tensión. Suele facilitar esta maniobra la aplicación inicial de los puntos sin anudar y luego ir anudándolos cuando se ha distribuido el tejido cómodamente.

Aunque las diferencias observadas en el grupo control en estudios retrospectivos no han sido estadísticamente significativas, la frecuencia de complicaciones locales es menor en las series en las que se aplicaron suturas de tensión progresiva para el cierre de los colgajos (12).

El tratamiento de este tipo de pseudoquistes consiste en su exéresis y en el cierre de la pared abdominal minimizando espacios muertos (13). Los tubos de drenaje deben mantenerse mientras sean productivos, empleándose una prenda compresiva durante las primeras semanas del postoperatorio.

\section{Conclusiones}

La formación de pseudoquistes de Morel-Lavallée es una complicación poco frecuente que puede aparecer en dermolipectomía abdominal sin lipoaspiración asociada, incluso varios años después de la intervención inicial.
Para prevenir esta complicación, el cierre de los colgajos cutáneos debe efectuarse minimizando espacios muertos y evitando deslizamientos entre la fascia muscular y el tejido subcutáneo.

La ecografía y la resonancia nuclear magnética permiten un diagnóstico de sospecha mediante visualización de formaciones encapsuladas, bien delimitadas y de contenido heterogéneo y su tratamiento es la extirpación quirúrgica más cierre de la herida por planos con suturas de anclaje plano a plano.

\section{Agradecimientos}

Agradecemos a todo el equipo del Servicio de Cirugía Plástica y Quemados del Hospital Universitario Virgen de la Arrixaca y al Dr. J. I. Guzmán Salinas su indispensable colaboración en este trabajo.

\section{Dirección del autor}

Dr. Álvaro Fernández García

C/ Mariano Estrada Lorca $n^{\circ} 15$, Portal 2, Piso $1^{\circ} \mathrm{D}$.

El Palmar. Murcia C.P: 30120. España.

e-mail: alvaro.fernandez@telefonica.net

\section{Bibliografía}

1. Flageul, G., Elbaz, J.S., Karcenty, B.: "Complications of plastic surgery of the abdomen”. Ann. Chir. Plast. Esthet. 1991, 44(4):497.

2. Stewart, K.J., Stewart, D.A., Coghlan, B., Harrison, D.H., Jones, B.M., Waterhouse, N.: "Complications of 278 consecutive abdominoplasties". J. Plast. Reconstr. Aesthet. Surg.; 2006, 59(11):1152.

3. Kim, J., Stevenson, T. R.: "Abdominoplasty, liposuction of the flanks, and obesity: analyzing risk factors for seroma formation". Plast. Reconstr Surg. 2006, 117(3):773. Discussion 780.

4. Zecha, P.J., Missotten, F.E.: "Pseudocyst formation after abdominoplasty extravasations of Morel.Lavallee". Br. J. Plast. Surg.; 19991, 52(6):500.

5. Ramírez OM.: "Abdominoplasty and abdominal wall rehabilitation: a comprehensive approach". Plast. Reconstr. Surg., 2000, 105 (1): 425 .

6. Broekhuysen, C. L., Nicolai, J.P.: "Formation of a pseudocyst following abdominoplasty". Ned. Tijdschr Geneeskd.2006,150(47):2610

7. Powers, M.L., Hatem, S.F., Sundaram, M.: "Morel-Lavallée lesion". Orthopedics. 2007, 30(4):250,322.

8. Tejwani, S.G., Cohen, S.B., Bradley, J.P.: "Management of Morel-Lavallée lesion of the knee: twenty-seven cases in the national football league". Am. J. Sports. Med. 2007, 35(7):1162.

9. Tsur, A., Galin, A., Kogan, L., Loberant, N.: "Morell-Lavallée syndrome after crush injury". Harefuah. 2006, 145(2):111.

10. Scaranelo, A.M., Davanco, R.A.: "Pseudocyst formation after abdominal liposuction extravasations of Morel-Lavallée on MR images". Br. J. Plast. Surg. 2005, 58(6):849.

11. Pollock, H., Pollock, T.: "Progressive tension sutures: a technique to reduce local complications in abdominoplasty". Plast. Reconstr. Surg. 2000, 105(7):2583.

12. Khan, S., Teotia, S.S., Mullis, W.F., Jacobs, W. E., Beasley, M.E., Smith, K.L., Eaves, F.F 3rd., Finical S.J., Watterson, P.A.: “Do progressive tension sutures really decrease complications in abdominoplasty?” Ann. Plast. Surg. 2006, 56(1):14. Discussion 20.

13. Roje, Z., Roje, Z., Karanovic, N., Utrobicic, I.: "Abdominoplasty complications: a comprehensive approach for the treatment of chronic seroma with pseudobursa". Aesthetic Plast. Surg. 2006, 30(5):611. 